\title{
Pathways leading to prevention of fatal and non-fatal cardiovascular disease: An interaction model on 15 years population- based cohort study
}

Najmeh Shakibaei ${ }^{1}$, Razieh Hassannejad ${ }^{2}$, Noushin Mohammadifard ${ }^{3}$, Hamid Reza Marateb ${ }^{4,5}$, Marjan Mansourian ${ }^{5,6^{*}}$, Miguel Angel Mañanas ${ }^{5,7}$ and Nizal Sarrafzadegan ${ }^{8,9}$

\begin{abstract}
Background: A comprehensive study on the interaction of cardiovascular disease (CVD) risk factors is critical to prevent cardiovascular events. The main focus of this study is thus to understand direct and indirect relationships between different CVD risk factors.

Methods: A longitudinal data on adults aged $\geq 35$ years, who were free of CVD at baseline, were used in this study. The endpoints were CVD events, whereas their measurements were demographic, lifestyle components, socioeconomics, anthropometric measures, laboratory findings, quality of life status, and psychological factors. A Bayesian structural equation modelling was used to determine the relationships among 21 relevant factors associated with total CVD, stroke, acute coronary syndrome (ACS), and fatal CVDs.

Results: In this study, a total of 3161 individuals with complete information were involved in the study. A total of 407 CVD events, with an average age of 54.77(10.66) years, occurred during follow-up. The causal associations between six latent variables were identified in the causal network for fatal and non-fatal CVDs. Lipid profile, with the coefficient of $0.26(0.01)$, influenced the occurrence of CVD events as the most critical factor, while it was indirectly mediated through risky behaviours and comorbidities. Lipid profile at baseline was influenced by a wide range of other protective factors, such as quality of life and healthy lifestyle components.

Conclusions: Analysing a causal network of risk factors revealed the flow of information in direct and indirect paths. It also determined predictors and demonstrated the utility of integrating multi-factor data in a complex framework to identify novel preventable pathways to reduce the risk of CVDs.
\end{abstract}

Keywords: Acute coronary syndrome, Bayesian approach, Cardiovascular disease, Stroke, Structural equation models

\footnotetext{
*Correspondence: j_mansourian@hlth.mui.ac.ir; marjan.mansourian@upc.edu

${ }^{5}$ Department of Automatic Control, Biomedical Engineering Research Center,

Universitat Politècnica de Catalunya, BarcelonaTech (UPC), Barcelona, Spain

${ }^{6}$ Pediatric Cardiovascular Research Center, Cardiovascular Research Institute,

Isfahan University of Medical Sciences, Isfahan, Iran

Full list of author information is available at the end of the article
}

C C The Author(s). 2020 Open Access This article is licensed under a Creative Commons Attribution 4.0 International License, which permits use, sharing, adaptation, distribution and reproduction in any medium or format, as long as you give appropriate credit to the original author(s) and the source, provide a link to the Creative Commons licence, and indicate if changes were made. The images or other third party material in this article are included in the article's Creative Commons licence, unless indicated otherwise in a credit line to the material. If material is not included in the article's Creative Commons licence and your intended use is not permitted by statutory regulation or exceeds the permitted use, you will need to obtain permission directly from the copyright holder. To view a copy of this licence, visit http://creativecommons.org/licenses/by/4.0/ The Creative Commons Public Domain Dedication waiver (http://creativecommons.org/publicdomain/zero/1.0/) applies to the data made available in this article, unless otherwise stated in a credit line to the data. 


\section{Background}

Cardiovascular disease (CVD), which is responsible for 17.9 million deaths, has been identified as the world's leading cause of death $[1,2]$. The World Health Organization (WHO) estimates that this number increases to 23.6 million by 2030 [3]. In low- and middleincome countries, more than three-quarters of deaths occur due to CVD, which results in the imposition of $80 \%$ of the global burden of CVD. Iran has a similar situation in which the burden of CVD will be more significant in the upcoming years $[4,5]$. However, most of the risk factors of this non-communicable disease (NCD) are "preventable" [6]. Therefore, the global strategy is to reduce the early incidence of CVD. The prevention of CVD can be four times more effective than subsequent measures [7]. Identifying relevant risk factors is the best way to correct the risky behaviours that make a person vulnerable to disease [8]. The interaction of irreversible factors (e.g., age, sex, family history of early CVD and race), behavioural factors (e.g., physical activity, smoking, and diet), and physiological risk factors (e.g., diabetes mellitus (DM), blood pressure (BP), obesity, and dyslipidaemia) may cause CVDs [9-15].

Many studies examined CVD risk factors [16-21]. However, they cannot usually detect causal pathways between the associated factors and CVD. Some studies investigated cardiovascular risk factors using structural equation modelling [17-21]. Among which, Azizi et al. studied the effect of emotional distress, physical activity, and body mass index (BMI) on the link between CVD and short sleep duration $(<7 \mathrm{~h}$ per day) [17]. ScottStorey et al. studied the severity of women's abuse that might affect women's risk of CVD providing preliminary evidence to support many hypothesised pathways using the severity of abuse [18]. Fong and Ho used Bayesian structural equation modelling (BSEM) to identify the association between depression scale, and the hospital anxiety a sample of 312 Chinese patients. It showed that the two-factor structure represents the appropriate scale of hospital depression and anxiety in clinical practice [19]. Kerkhof et al. presented SEM with different pathways indicating fat mass is influenced by atherosclerosis risk factors at the age of 21 years [20]. Goong et al. indicated that health behaviour modification performance could be described by health belief, social support, and changing the knowledge and attitude about health using the SEM in patients with CVDs [21].

Many CVD factors have been identified in previous studies, but it is not clear whether these factors influence CVDs directly or indirectly. Meanwhile, CVDs are chronic diseases influenced by various factors. Therefore, it is essential to study the multidimensional factors at the same time leading to CVD. To the best of our knowledge, no such multidimensional study approaches have been applied to the problem of different lifestyle components, laboratory factors, and comorbidities, at the same time, with indirect and direct effects. In this study, a series of BSEMs to examine the associations between a large number of risk factors and risk for total CVDs and its different categories were developed. By understanding the contribution of these factors leading CVDs, the findings of this study could be of great help for healthcare professionals in recognising strong predictors for applying effective strategies for CVDs prevention.

\section{Materials and methods \\ Study population}

Isfahan cohort study (ICS), a population-based study, has been performed by Isfahan Cardiovascular Research Center (ICRC), a WHO-collaborating centre (https:// apps.who.int/whocc/Detail.aspx?9Dsb+Z/ZGeLpVAy$\mathrm{gEBL}+\mathrm{Hg}==)$. It was designed to identify the effect of different risk factors on the incidence of CVD in a representative sample from the centre of Iran, including Isfahan, Arak, and Najafabad. Participants were selected using multistage cluster random sampling based on gender, age, and residence status (urban/rural) distribution. They were recruited from 2001 and were followed up every 2 years for major cardiovascular events and every 5 -years for measuring risk factors. Inclusion criteria included (1) being at least 35 years old without CVD and mental retardation diseases, (2) having Iranian nationality, (3) living in one of the above three cities for at least 6 months, and (4) not being pregnant [22]. This study is the secondary analysis of the ICS, which had the power of $90 \%$ to detect new CVD cases [22].

\section{Risk factors measurements}

Trained health professionals collected demographic, behavioural, socioeconomic characteristics, smoking, nutrition, and physical activity status. Smoking status was classified as non-smokers, ex-smokers, and smokers. Smokers were who smoked at least one cigarette a day while individuals who smoked before but did not smoke at the time of the interview (i.e., those who replied "no" to the question "are you smoking now?") were defined as ex-smokers. Fasting blood samples $(10 \mathrm{ml})$ were taken from participants to measure triglycerides (TG), lowdensity lipoproteins (LDL-C), high-density lipoproteins (HDL-C), and total cholesterol (Tcho). Diabetes was considered if fast blood sugar (FBS) $\geq 126 \mathrm{mg} / \mathrm{dL}$ or taking hypoglycaemic agents [23]. Dyslipidaemia was defined if $\mathrm{HDL}-\mathrm{C} \leq 50 \mathrm{mg} / \mathrm{dL}$ in women, and $\mathrm{HDL}-\mathrm{C} \leq 40 \mathrm{mg} / \mathrm{dL}$ in men, Tcho $\geq 200 \mathrm{mg} / \mathrm{dL}, \mathrm{TG} \geq 150 \mathrm{mg} / \mathrm{dL}$, and LDL-C $\geq$ $130 \mathrm{mg} / \mathrm{dL}$. The anthropometric parameters weight, height, waist circumference (WC), and hip circumference (HC) were measured by standard protocols. Obesity was considered as body mass index $(\mathrm{BMI}) \geq 30 \mathrm{~kg} / \mathrm{m}^{2}$. 
Abdominal obesity was defined as $\mathrm{HC} \geq 85.5 \mathrm{~cm}$ in women and $\mathrm{HC} \geq 91.5 \mathrm{~cm}$ in men, and high WC was defined as $W C \geq 94 \mathrm{~cm}$ in men or $W C \geq 80 \mathrm{~cm}$ in women $[24,25]$. Trained professionals measured systolic and diastolic blood pressure with standard protocol. According to the WHO definition, people with diastolic blood pressure $(\mathrm{DBP}) \geq 90$ or systolic blood pressure (SBP) $\geq 140 \mathrm{mmHg}$ or those using hypertensive drug users were considered as hypertensive patients [26].

\section{Instruments and questionnaires}

Dietary behaviours were assessed by a validated food frequency questionnaire (FFQ) [27]. Factor analysis was used to identify four main dietary patterns. The Mediterranean diet pattern was considered as the CVD mortality protective regime (i.e., healthy diet behaviour), while the other diet patterns were considered as an unhealthy diet behaviour [14]. A validated international physical activity questionnaire (IPAQ) was used to assess physical activity [28]. Depression and anxiety were detected using A general health questionnaire (GHQ-28) [29].

The quality of life was assessed using the $\mathrm{WHO}$ quality of life questionnaire (WHOQOL-BREF), including 26 questions, four subscales, and an overall score. Four areas of QOL were environmental health, social relations, mental, and physical health. Transportation, physical environment (weather, traffic, noise, and pollution), home environment, quality of social care, security, health and access, freedom, and financial resources were considered for environmental health. Sexual activities, social support, and personal relationships were considered for social relations. Mental health, on the other hand, consisted of focus, memory, learning, thinking, personal beliefs, spirituality or religion, self-esteem, positive feelings, and negative emotions. Work capacity, rest, sleep, discomfort, pain, mobility, fatigue, energy, medical assistance, drug dependency, and activities of daily living were considered for physical health [30-33].

\section{Outcomes}

Total CVD included significant events such as fatal and non-fatal myocardial infarction (MI), unstable angina (UA), sudden cardiac death (SCD), and fatal and nonfatal stroke. Two separate groups of specialists, each comprising four neurologists and cardiologists, reviewed all patient records (original questionnaires, secondary interviews, medical records, verbal autopsy, or death certificates). Then, the final decision was made on cardiovascular events. Ischemic heart disease (IHD) included MI, sudden cardiac death, and definitive or probable UA. The diagnosis of stroke was based on the WHO definition as a permanent neurological disorder for at least $24 \mathrm{~h}$. Acute coronary syndrome included all events of CVDs except stroke and sudden cardiac death.
Fatal CVD included fatal strokes, fatal MI, and sudden cardiac death [22]. Total CVD, stroke, ACS, and fatal CVD were separately considered in different risk factors' causal networks.

\section{Statistical analysis}

Continuous variables were represented by the mean and standard deviation (SD), while a number and percentage were used for the categorical variables. Comparisons in groups were obtained using logistic regression. Analyses were performed with STATA 14 (StataCorp. 2015. Stata Statistical Software: Release 14. College Station, TX: StataCorp LP.) and open BUGS version 3.2.3. A pairwise correlation test was used to produce the correlation matrix for each of the variables known or hypothesised to influence the CVD in adults.

\section{Modelling description}

SEM with a mixture of continuous and dichotomous variables was used to identify factors associated with fatal CVD, ACS, stroke, and total CVD. It was used to measure latent concepts such as healthy, risky behaviour or quality of life, which were not directly measured, as well as to explore the pattern of both direct and indirect relationships between the factors associated with the CVDs [34]. Due to a large number of explanatory variables, the Bayesian SEM was applied. In a Bayesian method, which is a broad approach to deal with complex situations, the useful prior information is used, and reliable results with small or medium sample size can be presented. The Bayesian approach is also used as an attractive method to generalise basic SEMs such as nonlinear, multilevel, and longitudinal SEMs [35]. We implement Markov Monte Carlo Chain Methods $(\mathrm{MCMC})$, and observations were obtained by Gibbs sampling on conditional density in open BUGs (Supplementary Materials 1, and 2, Additional file 1).

As shown in Fig. 1, the latent variables considered in this study were lipids, anthropometric, risky behaviour, comorbidities, quality of life, and healthy lifestyle component. Figure 1 contains 21 indicator variables and 6 exogenous latent variables and one endogenous latent variable.

The structural equation for CVD is shown as follows:

$$
\begin{aligned}
& \text { Lipids }+\gamma_{2} \times \text { Anthropometric }+\gamma_{3} \\
& \quad \times \text { Risky behavior }+\gamma_{4} \times \text { Comorbidities }+\gamma_{5} \\
& \quad \times \text { Quality of life }+\gamma_{6} \\
& \quad \times \text { Healthy lifestyle component }+\delta_{i} ;
\end{aligned}
$$

where $\gamma_{i}$ is the impact coefficient of latent variables and $\delta_{\mathrm{i}}$ is the random vector of the residuals. 


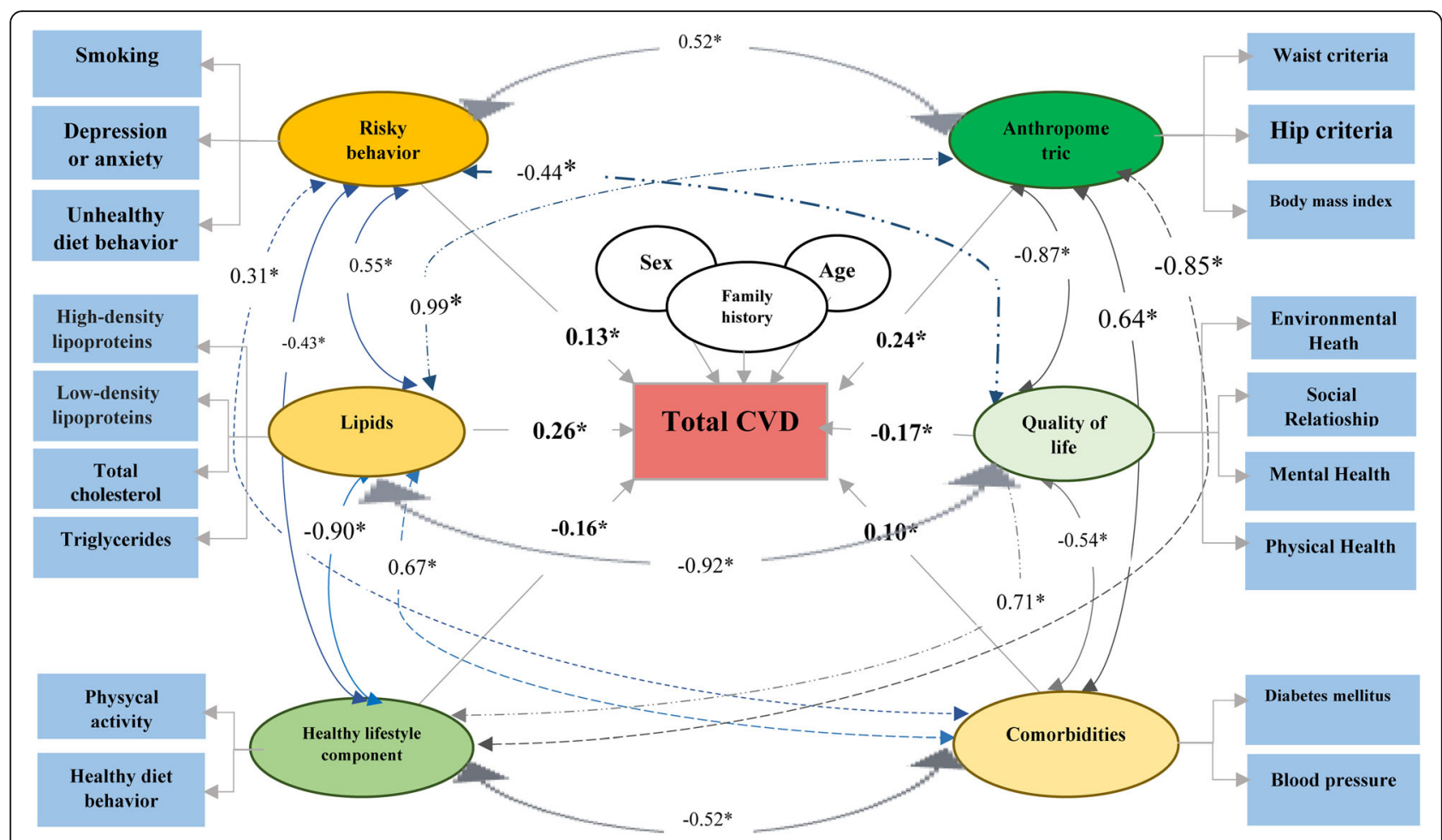

Fig. 1 BSEM the mediating effects of factor associated with total CVD in the Isfahan Cohort Study

\section{Result}

\section{Descriptive statistics}

Factor analysis was used to obtain a nutritional pattern. Four main dietary patterns were identified, including: "fast food", "animal fat", "Mediterranean", and "western". The "fast food diet" contained lots of carbonated drinks, sweets, pizzas, sausages, and burgers, while the "animal fat diet" was described by a high intake of meat, butter, whole milk, and cream. The consumption of olive oil, vegetables, fish, poultry, fruits, and non-hydrogenated vegetable oils (NHVO) were associated with the Mediterranean diet. The "western diet" included rice, pickles, red meat, hydrogenated vegetable oils, legumes, potatoes, and fried foods (Supplementary Table 1, Additional file 1).

In this study, from a total number of 3161 people, 180(0.05\%) men and 227(0.07\%) women with total CVD were observed. CVD-related risk factors, including lipids (LDL-C, TG, Tcho), anthropometric (HC, WC, BMI), risky behaviour (depression or anxiety, unhealthy diet behaviour, smoking), and comorbidities (BP, DM), were higher than those without CVD (Tables 1, and 2). The CVD group's healthy diet and physical activity, on the other hand, were lower than non-CVD subjects. It is also worth noting that a higher score in Tables 1 , and 2 shows a lower quality of life. Patients with CVD had a lower quality of life compared with others.

The lipid profiles (HDL-C, LDL-C, total Cho) have the highest impact on CVD. Other significant variables were anthropometric indices (WC, and $\mathrm{HC}$, and $\mathrm{BMI}$ ), risky behaviour (depression or anxiety, unhealthy diet behaviour, and smoking status), comorbidities (DM, BP), quality of life (environmental health, social relationship, mental health, physical health), and healthy lifestyle components (physical activity and healthy diet behaviour) (Fig. 1). Thus, the higher the level of lipid profiles, the higher the risk of CVDs. Also, the association between higher quality of life and a healthy lifestyle with CVDs incidence was significantly negative.

Applying the BSEM, a causal network is plotted using different exploratory factors, twenty-one measurements of CVD risk factors at baseline, including cardiovascular risk factors. Models obtained in this study classified these variables into six latent variables (Table 3). Also, in line with this study, models for stroke, ACS, and fatal CVD (Tables 4 and 5) were developed. The most important finding of this study based on the comparison of three models of stroke, acute coronary syndrome, and fatal CVD, is the significant effect of risky behaviour on these subtypes of CVD (Stroke (0.14), ACS (0.20), Fatal CVD (0.11)). Other direct and indirect relationships of all three models are presented in Tables 4 and 5. It should be noted that all relationships were significant except for a family history of CVD.

Convergence statistical test was performed for all parameters, and the Goleman-Robin test statistic was close to one. The Monte Carlo errors were also very low for 
Table 1 Characteristics of study participants for CVD and Stroke

\begin{tabular}{|c|c|c|c|c|c|c|}
\hline \multirow[t]{2}{*}{ Characteristics } & \multicolumn{2}{|l|}{ CVD } & \multirow[t]{2}{*}{$P$-value } & \multicolumn{2}{|l|}{ Stroke } & \multirow[t]{2}{*}{$P$-value } \\
\hline & $\begin{array}{l}\text { No } \\
N=2754\end{array}$ & $\begin{array}{l}\text { Yes } \\
N=407\end{array}$ & & $\begin{array}{l}\mathrm{No} \\
N=3084\end{array}$ & $\begin{array}{l}\text { Yes } \\
N=77\end{array}$ & \\
\hline \multicolumn{7}{|l|}{ Demographic Variables } \\
\hline Age (years) & $48.39(9.96)$ & $54.77(10.66)$ & 0.00 & $49.04(10.19)$ & $56.08(11.33)$ & 0.18 \\
\hline Sex & & & 0.01 & & & 0.52 \\
\hline Man & 1438 (52.21\%) & 180 (44.22\%) & & $1583(51.3 \%)$ & $35(45.5 \%)$ & \\
\hline Woman & 1316 (47.79\%) & $227(55.78 \%)$ & & $1501(48.7 \%)$ & $42(54.5 \%)$ & \\
\hline Family History of CVD & & & & & & 0.15 \\
\hline Yes & $152(5.50 \%)$ & $34(8.4 \%)$ & & $179(5.8 \%)$ & 7 (9.1\%) & \\
\hline No & $2602(94.50 \%)$ & $373(91.6 \%)$ & & 2905 (94.2\%) & 70 (90.9\%) & \\
\hline \multicolumn{7}{|l|}{ Lipids } \\
\hline $\mathrm{HDL}(\mathrm{mg} / \mathrm{dL})$ & $46.88(10.29)$ & $46.71(10.57)$ & 0.76 & $46.85(10.32)$ & $47.7(10.67)$ & 0.85 \\
\hline LDL (mg/dL) & 127.66 (42.89) & $135.81(44.87)$ & 0.00 & $128.67(43.23)$ & $130.3(43.33)$ & 0.74 \\
\hline $\mathrm{TG}(\mathrm{mg} / \mathrm{dL})$ & 186.58 (99.77) & $222.22(117.27)$ & 0.00 & 190.46 (102.34) & 218.99 (119.57) & 0.04 \\
\hline TChol (mg/dL) & $211.86(51.37)$ & $226.97(55.06)$ & 0.00 & $213.62(52.06)$ & $221.18(55.36)$ & 0.20 \\
\hline \multicolumn{7}{|l|}{ Anthropometric } \\
\hline $\mathrm{HC}(\mathrm{cm})$ & $101.99(9.51)$ & $102.63(9.64)$ & 0.20 & $102.03(9.53)$ & $103.95(9.41)$ & 0.08 \\
\hline WC (cm) & $94.52(11.90)$ & $97.86(12.43)$ & 0.00 & $94.87(12.00)$ & $98.27(12.27)$ & 0.19 \\
\hline BMI $\left(\mathrm{kg} / \mathrm{m}^{2}\right)$ & $26.82(4.33)$ & $27.41(4.64)$ & 0.01 & $26.87(4.35)$ & $28.08(5.10)$ & 0.04 \\
\hline \multicolumn{7}{|l|}{ Risky behaviour } \\
\hline Depression or anxiety & $1.97(2.19)$ & $2.44(2.69)$ & 0.00 & $2.03(2.25)$ & $2.44(2.84)$ & 0.21 \\
\hline Unhealthy diet behaviour & $2.12(2.46)$ & $1.71(2.13)$ & 0.00 & $2.08(2.42)$ & $1.81(2.54)$ & 0.36 \\
\hline Smoking Status & & & 0.00 & & & 0.01 \\
\hline Smoker & $414(15.03 \%)$ & $81(19.90 \%)$ & & $477(15.5 \%)$ & $18(23.4 \%)$ & \\
\hline Ex-Smoker & $154(5.59 \%)$ & $32(7.86 \%)$ & & $180(5.8 \%)$ & $6(7.8 \%)$ & \\
\hline Non-Smoker & $2186(79.37 \%)$ & $294(72.23 \%)$ & & $2427(78.7 \%)$ & $53(68.8 \%)$ & \\
\hline \multicolumn{7}{|l|}{ Comorbidities } \\
\hline $\mathrm{BP}$ & & & 0.46 & & & 0.00 \\
\hline Yes & $660(24 \%)$ & 179 (44\%) & & $796(25.8 \%)$ & $43(55.8 \%)$ & \\
\hline No & $2094(76 \%)$ & $228(56 \%)$ & & $2288(74.2 \%)$ & $34(44.2 \%)$ & \\
\hline DM & & & 0.00 & & & 0.60 \\
\hline Yes & $227(8.2 \%)$ & $97(23.8 \%)$ & & $305(9.9 \%)$ & $19(24.7 \%)$ & \\
\hline No & $2527(91.8 \%)$ & $310(76.2 \%)$ & & $2779(90.1 \%)$ & $58(75.3 \%)$ & \\
\hline \multicolumn{7}{|l|}{ Quality of life } \\
\hline Environmental Health & $32.76(17.02)$ & $39.47(16.67)$ & 0.00 & $33.37(17.07)$ & $43.48(16.67)$ & 0.00 \\
\hline Social Relationship & $33.92(15.72)$ & $36.40(16.16)$ & 0.00 & $34.12(15.75)$ & $38.87(17.00)$ & 0.01 \\
\hline Mental Health & $28.14(18.62)$ & $29.88(19.22)$ & 0.08 & $28.24(18.66)$ & $33.49(19.70)$ & 0.01 \\
\hline Physical Health & $34.45(16.63)$ & $37.00(16.25)$ & 0.00 & $34.63(16.61)$ & $41.00(14.90)$ & 0.00 \\
\hline \multicolumn{7}{|l|}{ Healthy lifestyle component } \\
\hline Healthy diet & $2.33(3.09)$ & $1.83(2.41)$ & 0.00 & $2.28(3.02)$ & $1.83(2.70)$ & 0.19 \\
\hline Daily physical activity (METs-min/day) & $910.65(539.56)$ & $839.50(562.06)$ & 0.01 & $904.11(543.70)$ & $796.63(503.70)$ & 0.08 \\
\hline
\end{tabular}

Abbreviations: cardiovascular disease, HDL high-density lipoproteins-cholesterol, $L D L$ low-density lipoproteins -cholesterol, $T G$ triglycerides, $T C$ total cholesterol, $H C$ hip circumference, WC Waist circumference, $B M I$ Body mass index, $B P$ blood pressure, $D M$ diabetes mellitus 
Table 2 Characteristics of study participants for models (Acute coronary disease and Fatal CVD)

\begin{tabular}{|c|c|c|c|c|c|c|}
\hline \multirow[t]{2}{*}{ Characteristics } & \multicolumn{2}{|l|}{ ACS } & \multirow{2}{*}{$\begin{array}{l}P \text { - } \\
\text { value }\end{array}$} & \multicolumn{2}{|l|}{ Fatal CVD } & \multirow{2}{*}{$\begin{array}{l}P \text { - } \\
\text { value }\end{array}$} \\
\hline & $\begin{array}{l}\text { No } \\
N=2846\end{array}$ & $\begin{array}{l}\text { Yes } \\
N=315\end{array}$ & & $\begin{array}{l}\text { No } \\
N=2979\end{array}$ & $\begin{array}{l}\text { Yes } \\
N=182\end{array}$ & \\
\hline \multicolumn{7}{|l|}{ Demographic variables } \\
\hline Age (years) & $48.39(9.9)$ & $53.9(10.3)$ & 0.00 & $48.3(9.9)$ & $55.8(11.5)$ & 0.00 \\
\hline \multicolumn{7}{|l|}{ Sex } \\
\hline Man & 1478 (51.53\%) & 140 (44.44\%) & 0.00 & $1550(52.03 \%)$ & 69 (37.91\%) & 0.00 \\
\hline Woman & $1368(48.47 \%)$ & $175(55.56 \%)$ & & 1429 (47.97\%) & 113 (62.09\%) & \\
\hline \multicolumn{7}{|l|}{ Family History of CVD } \\
\hline Yes & $174(6.11 \%)$ & $27(8.60 \%)$ & 0.00 & $264(8.80 \%)$ & $11(6.04 \%)$ & 0.56 \\
\hline No & 2672 (93.89\%) & $288(91.40 \%)$ & & 2715 (91.20\%) & 171 (93.96\%) & \\
\hline \multicolumn{7}{|l|}{ Lipids } \\
\hline $\mathrm{HDL}(\mathrm{mg} / \mathrm{dL})$ & $46.88(10.29)$ & $46.65(10.54)$ & 0.7 & $46.8(10.2)$ & $46.0(10.2)$ & 0.29 \\
\hline $\mathrm{LDL}(\mathrm{mg} / \mathrm{dL})$ & $127.66(42.89)$ & $138.01(45.6)$ & 0.00 & $127.6(42.8)$ & $133.6(45.3)$ & 0.06 \\
\hline $\mathrm{TG}(\mathrm{mg} / \mathrm{dL})$ & $186.5(99.77)$ & $223.8(117.8)$ & 0.00 & $186.5(99.7)$ & 217 (113.3) & 0.00 \\
\hline TChol (mg/dL) & $211.86(51.3)$ & $229.4(56.1)$ & 0.00 & $211.8(51.3)$ & $223.2(53.8)$ & 0.00 \\
\hline \multicolumn{7}{|l|}{ Anthropometric } \\
\hline $\mathrm{HC}(\mathrm{cm})$ & $101.9(9.5)$ & $102.5(9.7)$ & 0.36 & $101.9(9.5)$ & $101.8(0.2)$ & 0.85 \\
\hline WC $(\mathrm{cm})$ & $94.5(11.9)$ & $97.81(12.5)$ & 0.00 & 94.5 (11.9) & $97.0(13.1)$ & 0.01 \\
\hline $\mathrm{BMI}\left(\mathrm{kg} / \mathrm{m}^{2}\right)$ & $26.82(4.33)$ & $27.34(11.94)$ & 0.04 & $26.8(4.33)$ & $27.2(4.8)$ & 0.15 \\
\hline \multicolumn{7}{|l|}{ Risky behaviour } \\
\hline depression or anxiety & $1.97(2.1)$ & $2.4(2.6)$ & 0.00 & $1.9(2.1)$ & $2.5(2.7)$ & 0.01 \\
\hline unhealthy diet behaviour & $2.1(2.4)$ & $1.7(2.0)$ & 0.00 & $2.1(2.4)$ & $1.7(2.0)$ & 0.02 \\
\hline Smoking Status & & & 0.01 & & & 0.00 \\
\hline Smoker & $434(15.25 \%)$ & $61(19.37 \%)$ & & $489(16.41 \%)$ & $45(24.73 \%)$ & \\
\hline Ex-Smoker & $161(5.67 \%)$ & $25(7.94 \%)$ & & $229(7.68 \%)$ & $12(6.60 \%)$ & \\
\hline Non-Smoker & $2251(79.08 \%)$ & $229(72.69 \%)$ & & $2261(75.91 \%)$ & $125(68.67 \%)$ & \\
\hline \multicolumn{7}{|l|}{ Comorbidities } \\
\hline \multicolumn{7}{|l|}{ BP } \\
\hline Yes & 709 (24.91\%) & $132(41.90 \%)$ & 0.63 & 772 (25.91\%) & $81(44.51 \%)$ & 0.05 \\
\hline No & 2137 (75.08\%) & $183(58.10 \%)$ & & 2207 (74.09\%) & $101(55.49 \%)$ & \\
\hline \multicolumn{7}{|l|}{ DM } \\
\hline Yes & $255(8.96 \%)$ & $74(23.50 \%)$ & 0.00 & $339(11.37 \%)$ & $45(24.73 \%)$ & 0.001 \\
\hline No & 2591 (91.04\%) & $241(76.50 \%)$ & & $2640(88.63 \%)$ & $137(75.27 \%)$ & \\
\hline \multicolumn{7}{|l|}{ Quality of life } \\
\hline Environmental Health & $32.7(17.0)$ & $38.0(16.4)$ & 0.00 & $32.7(17.0)$ & $40.6(16.5)$ & 0.00 \\
\hline Social Relationship & $33.9(15.7)$ & $35.1(15.8)$ & 0.18 & $33.9(15.7)$ & $36.9(15.9)$ & 0.01 \\
\hline Mental Health & $28.1(18.6)$ & $28.3(19.0)$ & 0.84 & $28.1(18.6)$ & $31.4(18.5)$ & 0.02 \\
\hline Physical Health & $34.4(16.6)$ & $35.5(16.5)$ & 0.2 & $34.4(16.6)$ & $38.3(15.9)$ & 0.00 \\
\hline \multicolumn{7}{|l|}{ Healthy lifestyle component } \\
\hline Healthy diet & $2.3(3.0)$ & $1.8(2.3)$ & 0.00 & $2.3(3.0)$ & $1.9(2.4)$ & 0.13 \\
\hline Daily physical activity (METs-min/day) & $910.6(539.5)$ & $851.0(571.3)$ & 0.006 & $910.6(539.5)$ & $828.3(521.1)$ & 0.04 \\
\hline
\end{tabular}


Table 3 Estimated direct, indirect effects related factor for CVD

\begin{tabular}{|c|c|c|c|c|c|c|c|}
\hline \multicolumn{8}{|l|}{ Type of effect } \\
\hline \multicolumn{4}{|l|}{ Direct effect } & \multicolumn{4}{|l|}{ Indirect effect } \\
\hline & Coefficient & SD & $95 \% \mathrm{Cl}$ & & Coefficient & SD & $95 \% \mathrm{Cl}$ \\
\hline \multirow[t]{4}{*}{ Age- > total CVD } & \multirow[t]{4}{*}{0.002} & \multirow[t]{4}{*}{0.0008} & \multirow[t]{4}{*}{$(0.001,0.004)$} & Lipids- > HDL & 1 & & \\
\hline & & & & Lipids- > LDL & 0.25 & 0.007 & $(0.22,0.26)$ \\
\hline & & & & Lipids- > Tgd & 0.84 & 0.02 & $(0.81,0.88)$ \\
\hline & & & & Lipids- > Cho & 0.47 & 0.01 & $(0.44,0.50)$ \\
\hline \multirow[t]{3}{*}{ Family history - > total CVD } & \multirow[t]{3}{*}{0.000} & \multirow[t]{3}{*}{0.0006} & \multirow{3}{*}{$\begin{array}{l}(-0.001 \\
0.0009)\end{array}$} & Anthropometric- > HC & 1 & & \\
\hline & & & & Anthropometric- > WC & 0.78 & 0.006 & $(0.77,0.80)$ \\
\hline & & & & Anthropometric- > BMI & 0.10 & 0.01 & $(0.08,0.11)$ \\
\hline \multirow[t]{3}{*}{ Sex - > total CVD } & \multirow[t]{3}{*}{0.004} & \multirow[t]{3}{*}{0.001} & \multirow[t]{3}{*}{$(0.007,0.012)$} & Unhealthy life style- > smoking & 1 & & \\
\hline & & & & Risky behaviour - > depression and anxiety & 0.79 & 0.1 & $(0.42,1.22)$ \\
\hline & & & & Risky behaviour - > unhealthy diet behaviour & -0.57 & 0.2 & $\begin{array}{l}(-0.99,- \\
0.16)\end{array}$ \\
\hline \multirow[t]{2}{*}{ Lipids - > total CVD } & \multirow[t]{2}{*}{0.26} & \multirow[t]{2}{*}{0.01} & \multirow[t]{2}{*}{$(0.26,0.28)$} & Comorbidities- > DM & 1 & & \\
\hline & & & & Comorbidities- > BP & 0.74 & 0.006 & $(0.73,0.75)$ \\
\hline \multirow[t]{4}{*}{ Anthropometric - > total CVD } & \multirow[t]{4}{*}{0.24} & \multirow[t]{4}{*}{0.01} & \multirow[t]{4}{*}{$(0.22,0.26)$} & Quality of life- > Environmental Heath & 1 & & \\
\hline & & & & Quality of life- > Social Relationship & 0.45 & 0.004 & $(0.44,0.47)$ \\
\hline & & & & Quality of life- > Mental Health & 0.52 & 0.005 & $(0.51,0.54)$ \\
\hline & & & & Quality of life- > Physical Health & 0.53 & 0.004 & $(0.51,0.54)$ \\
\hline \multirow[t]{2}{*}{ risky behaviour-> total CVD } & \multirow[t]{2}{*}{0.13} & \multirow[t]{2}{*}{0.03} & \multirow[t]{2}{*}{$(0.10,0.14)$} & $\begin{array}{l}\text { Healthy lifestyle component- > physical } \\
\text { activity }\end{array}$ & 1 & & \\
\hline & & & & $\begin{array}{l}\text { Healthy lifestyle component- > Healthy diet } \\
\text { behaviour }\end{array}$ & 0.99 & 0.01 & $(0.95,1.03)$ \\
\hline Comorbidities - > total CVD & 0.10 & 0.001 & $(0.08,0.11)$ & Lipids $<->$ Anthropometric & 0.99 & 0.15 & $(0.84,1.49)$ \\
\hline & & & & Lipids $<->$ Risky behaviour & 0.55 & 0.13 & $(0.22,0.80)$ \\
\hline & & & & Lipids $<->$ Comorbidities & 0.67 & 0.08 & $(0.53,0.89)$ \\
\hline & & & & Lipids $<->$ Quality of life & -0.92 & 0.12 & $\begin{array}{l}(-1.18, \\
-0.71)\end{array}$ \\
\hline & & & & Lipids $<->$ healthy lifestyle component & -0.90 & 0.12 & $\begin{array}{l}(-1.16,- \\
0.70)\end{array}$ \\
\hline Quality of life - > total CVD & -0.17 & 0.01 & $(-0.18,-$ & Anthropometric $<->$ Risky behaviour & 0.52 & 0.12 & $(0.22,0.75)$ \\
\hline & & & & Anthropometric $<->$ Comorbidities & 0.64 & 0.09 & $(0.50,0.85)$ \\
\hline & & & & Anthropometric $<->$ Quality of life & -0.87 & 0.12 & $\begin{array}{l}(-1.12,- \\
0.67)\end{array}$ \\
\hline & & & & $\begin{array}{l}\text { Anthropometric }<->\text { healthy lifestyle } \\
\text { component }\end{array}$ & -0.85 & 0.11 & $\begin{array}{l}(-1.11,- \\
0.67)\end{array}$ \\
\hline Healthy & -0.16 & 0.008 & $(-0.17,-$ & Risky behaviour $<->$ Comorbidities & 0.315 & 0.07 & $(0.13,0.45)$ \\
\hline lifestyle - > total CVD & & & & Risky behaviour $<->$ Quality of life & -0.44 & 0.10 & $\begin{array}{l}(-0.63,- \\
0.18)\end{array}$ \\
\hline & & & & $\begin{array}{l}\text { Risky behaviour }<->\text { healthy lifestyle } \\
\text { component }\end{array}$ & -0.43 & 0.10 & $\begin{array}{l}(-0.61,- \\
0.18)\end{array}$ \\
\hline & & & & $\begin{array}{l}\text { Comorbidities } \\
<->\text { Quality of life }\end{array}$ & -0.54 & 0.07 & $\begin{array}{l}(-0.72,- \\
0.43)\end{array}$ \\
\hline & & & & $\begin{array}{l}\text { Comorbidities } \\
<->\text { healthy lifestyle component }\end{array}$ & -0.52 & 0.07 & $\begin{array}{l}(-0.71,- \\
0.42)\end{array}$ \\
\hline & & & & Quality of life $<->$ healthy lifestyle component & 0.71 & 0.09 & $(0.56,0.92)$ \\
\hline
\end{tabular}

Abbreviations: cardiovascular disease, $H D L$ high-density lipoproteins-cholesterol, $L D L$ low-density lipoproteins -cholesterol, $T G$ triglycerides, $T C$ total cholesterol, $H C$ hip circumference, WC Waist circumference, BMI Body mass index, BP blood pressure, DM diabetes mellitus 
Table 4 Estimated direct effects related factor for models (Stroke, ACS, and Fatal CVD)

\begin{tabular}{llll}
\hline Models (Direct effect) & & & \\
\hline & $\begin{array}{l}\text { Stroke } \\
\text { Coefficients } \\
(S D)\end{array}$ & $\begin{array}{l}\text { ACS } \\
\text { Coefficients } \\
(\text { SD })\end{array}$ & $\begin{array}{l}\text { Fatal CVD } \\
\text { Coefficients } \\
(S D)\end{array}$ \\
\hline Age-> & 0.001 & 0.002 & 0.002 \\
& $(0.00008)$ & $(0.00005)$ & $(0.00006)$ \\
Family history $->$ & $0.000(0.002)$ & $0.000(0.002)$ & $0.00(0.003)$ \\
Sex $->$ & $0.01(0.003)$ & $0.009(0.002)$ & $0.009(0.003)$ \\
Lipids $->$ & $0.26(0.003)$ & $0.24(0.003)$ & $0.28(0.003)$ \\
Anthropometric $->$ & $0.23(0.002)$ & $0.23(0.002)$ & $0.25(0.002)$ \\
Risky behaviour $->$ & $0.14(0.007)$ & $0.20(0.02)$ & $0.11(0.01)$ \\
Comorbidities $->$ & $0.10(0.0003)$ & $0.11(0.0008)$ & $0.11(0.0007)$ \\
Quality of life $->$ & $-0.16(0.002)$ & $-0.15(0.001)$ & $-0.17(0.001)$ \\
Healthy lifestyle & $-0.15(0.001)$ & $-0.15(0.001)$ & $-0.17(0.001)$ \\
component $->$ & & & \\
\hline
\end{tabular}

the entire parameters in the models. Also, the goodness of fit of the models was assessed using Posterior Predictive $P$-value (P.P. $P$-value). Such values were $0.55,0.54$, 0.54, and 0.49 for CVD, stroke, ACS, and Fatal CVD, respectively (Supplementary Material 3 (A, B, and C), Additional file 1).

\section{Discussion}

In this study, BSEM was used to identify both direct and indirect pathways leading to total CVD, ACS, stroke, and fatal CVDs. Such an increased or decreased correlation between the traditional risk factors was related to the influence of the confounding variables while were adjusted by indirect pathways. Although previous studies considered limited risk factors, mostly in binary scale, this study showed preventive pathways with a large number of risk factors in both continuous and binary measurement scales. The Bayesian framework was applied for SEM modelling, which is reliable, especially in small sample size studies. Moreover, the resampling method used in MCMC was used in this study [36].

Many studies were performed in the literature on CVDs. Although the identification of CVD risk factors seems to be relatively comprehensive, the precise and indirect mechanisms of the associated factors underlying CVD remains unclear [37]. It is a critical gap since healthy behaviours lead to both reduced risk and more effective treatments $[17,38]$. This is the first study in the field of CVD, to the best of our knowledge, that extensively examined the direct and indirect effects of comprehensive cardiovascular-related factors using BSEM in a population-based cohort platform.

Although the indirect effects of family history of CVD, age, lipid profiles, anthropometric indices, healthy lifestyle, quality of life, high-risk behaviours, having hypertension and high blood sugar on CVDs were not covered in the literature, many studies discussed the direct effect of such factors in which these results are in line with [9, $11,12,15,38-50]$. Causal networks evaluate causal relationships among variables beyond partial correlations and thus play a fundamental step in risk prediction. For example, although many studies listed blood pressure as the most critical risk factor for CVD [51-55], this study

Table 5 Estimated indirect effects related factor for models (Stroke, ACS, and Fatal CVD)

\begin{tabular}{llll}
\hline Models (Indirect effect based on $\beta(S D))$ & & & \\
\hline & Stroke & ACS & Fatal CVD \\
& Coefficients (SD) & Coefficients (SD) & $0.98(0.1)$ \\
\hline Lipids $<->$ Anthropometric & $0.99(0.1)$ & $0.98(0.1)$ & $0.36(0.2)$ \\
Lipids $<->$ Risky behaviour & $0.60(0.1)$ & $0.65(0.4)$ & $0.72(0.08)$ \\
Lipids $<->$ Comorbidities & $0.67(0.08)$ & $0.64(0.07)$ & $-0.98(0.1)$ \\
Lipids $<->$ Quality of life & $-0.91(0.1)$ & $-0.86(0.09)$ & $-0.98(0.1)$ \\
Lipids $<->$ healthy lifestyle component & $-0.90(0.1)$ & $-0.85(0.09)$ & $0.34(0.2)$ \\
Anthropometric $<-$ Risky behaviour & $0.57(0.1)$ & $0.62(0.4)$ & $0.69(0.08)$ \\
Anthropometric $<->$ Comorbidities & $0.63(0.08)$ & $0.61(0.07)$ & $-0.93(0.1)$ \\
Anthropometric $<->$ Quality of life & $-0.85(0.1)$ & $-0.82(0.09)$ & $-0.93(0.1)$ \\
Anthropometric $<->$ healthy lifestyle component & $-0.84(0.1)$ & $-0.81(0.09)$ & $0.20(0.1)$ \\
Risky behaviour $<->$ Comorbidities & $0.32(0.07)$ & $-0.37(0.2)$ & $-0.28(0.1)$ \\
Risky behaviour $<->$ Quality of life & $-0.46(0.1)$ & $-0.51(0.3)$ & $-0.28(0.1)$ \\
Risky behaviour $<->$ healthy lifestyle component & $-0.45(0.1)$ & $-0.50(0.3)$ & $-0.56(0.06)$ \\
Comorbidities $<->$ Quality of life & $-0.53(0.07)$ & $-0.51(0.06)$ & $-0.57(0.09)$ \\
Comorbidities $<->$ healthy life style & $-0.53(0.07)$ & $-0.50(0.06)$ & \\
\hline
\end{tabular}


did not observe such a relationship. According to the results, CVD is mostly influenced by lipid profiles, which is in line with the CVD prevention study in Japan [56] and Brisighella heart study [57].

The proposed model indicated that the quality of life and healthy lifestyle components have the highest impact on lipid profile to prevent CVD. Therefore, individuals need to improve their quality of life indicators. Taking into account the physical health, as the first step, is essential. Indeed, in the absence of physical health, other indicators of quality of life do not improve. Environmental health was the second effective factor in these results. The social relationships with other people and also mental health improvement can play an essential role in CVD prevention [58, 59]. This study also highlighted the health benefits of high physical activity and having a healthy diet. Healthy lifestyle components also have a strong relationship with the quality of life variable. Therefore, considering the modifiable components of a healthy lifestyle, the healthy diet and the proper amount of physical activity (at least $150 \mathrm{~min}$ of physical activity per week [60]), are necessary to prevent CVD in adults [60, 61].

A healthy diet was promoted in the literature to prevent CVD [62]. The Mediterranean diet is one of the diet patterns in recent years, focusing on more consumption of plant foods (e.g., seeds, nuts, beans, fruits, and vegetables) and low to moderate amounts of animal food (e.g., red meat). In this diet, chicken, fish, vegetables, fruits, olive oil, NHVO, and fibres are mostly focused. Unhealthy diets, on the other hand, including western, animal fat, and fast food diets are characterised by various food items with high trans fatty acids (TFA), saturated fatty acid (SFA), and less monounsaturated fatty acid (MUFA) and Polyunsaturated fatty acid (PUFA). Such unhealthy diets also included rice, potato, and sweets, which contain high refined carbohydrates increase TG, and reduce HDL-C, as two essential components in the Iranian lipid profile [14, 62-64].

Obesity and overweight are general risk factors of CVD [48]. Due to the close relationship between lipid profiles and anthropometric indices, it is necessary to take into account the improved lifestyle and quality of life to reduce the incidence of CVD. Moreover, risky behaviour endangers health and quality of life and has a positive direct effect on anthropometric indices and lipid profile. Stress, on the other hand, can have immediate cardiovascular consequences, such as heart attack and sudden cardiac arrest, which can occur even in healthy people [65]. The possible role of smoking-related disorders in CVD should not be ignored either. Since stress and smoking are also modifiable factors, people can easily manage these high-risk behaviours to prevent CVDs $[55,66]$.
Non-communicable Disease Prevention organisations consider the unhealthy diet, physical inactivity, and alcohol use as common causes for stroke, heart disease, diabetes mellitus, cancer, and lung disease [67]. Also, GBD findings suggest that two-thirds of strokes occur among people under the age of 70, so they should no longer be considered as old-age diseases. It has also been reported that stroke is increasing in young and middle-aged adults, possibly due to increased metabolic risk factors such as obesity and diabetes mellitus. The main risk factors for stroke were smoking and high blood pressure. Other risk factors included obesity, high blood cholesterol, and diabetes mellitus [68]. In the proposed model, anthropometric indices, which were more critical than lipid profiles and risky behaviour, play a significant role in stroke.

Researches showed that the following risk factors increase the likelihood of ACS occurrence: old age, diabetes, hypertension, tobacco use, risky behaviour, male gender, and a family history of heart disease [69]. The results, on the other hand, highlighted the impact of risky behaviour that cannot be ignored. Rücker et al. stated that the main risk factors of fatal CVDs were total cholesterol, smoking, and also having higher systolic blood pressure [70]. Current results showed that the lipid profile was the most critical factor for (fatal) CVDs, emphasising the control of lipid profile.

In this study, a reliable statistical approach was used on a representative population of the Eastern and Mediterranean region (EMR), aiming to guide healthcare policymakers and practitioners on identifying risk factors and evidence-based screening of people at high risk of developing CVD (Fig. 1, Table 3). The lack of knowledge about preventable risk factors could hinder the action plans on reducing CVDs [71].

\section{Study strengths and limitations}

Having developed a series of BSEMs, it is possible to examine the associations between a large number of risk factors and risk for total CVD and its different categories. The findings of this study could be of great help for healthcare professionals in recognising strong predictors for applying effective strategies for CVDs prevention. However, this study ignored the possible effects of sleep disorders leading to dyslipidaemia and CVD. Also, the effect of environmental factors, such as air pollution indices, on CVDs incidence, was not taken into account. Moreover, time-varying covariates such as treatments were not considered since this study aimed to evaluate blood factors and lifestyle components.

\section{Conclusion}

The flow of information in indirect and direct paths was revealed by analysing a causal network of risk factors. It 
also determined predictors and suitable targets for intervention, such as decreasing lipid profile in the general population. Reducing the risk of CVDs could be achieved when multi-factor data is integrated into a complex framework by identifying novel preventable pathways. However, clinical trials are required for lipid profile adjustment as a controllable factor among adults, which could lead to a significant CVD risk reduction.

\section{Supplementary information}

Supplementary information accompanies this paper at https://doi.org/10. 1186/s12944-020-01375-8.

Additional file 1: The details of the statistical model and its validation. It includes the following: Supplementary Material 1. Modeling description. Supplementary Material 2. WinBUGS code BSEM

Supplementary Table 1. Factor loading matrix for dietary patterns. Supplementary Material 3. An example of Golman Robin test, convergence diagrams, and Monte Carlo error: A. Golman Robin test. B: Convergence diagrams for the first 1000 updates (trace plot). Table C. Monte Carlo error for total CVD

\section{Abbreviations}

CVD: Cardiovascular; ACS: Acute coronary syndrome; BSEM: Bayesian structural equation modeling; NCD: Non-communicable diseases; DM: Diabetes mellitus; BP: Blood pressure; ICS: Isfahan cohort study; AUC: Area under the curve; Tcho: Total cholesterol; HDL-C: High-density lipoproteins; LDL-C: Low-density lipoproteins; TG: Triglycerides; FBS: Fast blood sugar; HC: Hip criteria; BMI: Body mass index; WC: Waist criteria; SBP: Systolic blood pressure; DBP: Diastolic blood pressure; FFQ: Food frequency questionnaire; IPAQ: International physical activity questionnaire; GHQ: General health questionnaire; WHOQOL: World health organisation quality of life; MI: Myocardial infraction; SCD: Sudden cardiac death; UA: Unstable angina; IHD: Ischemic heart disease; SD: Standard deviation; SEM: Structural equation model; MCMC: Markov chain monte carlo; PP Pvalue: Posterior predictive $P$-value; NHVO: Non-hydrogenated vegetable oils; TFA: Trans fatty acids; SFA: Saturated fatty acid; MUFA: Monounsaturated fatty acid; PUFA: Polyunsaturated fatty acid; EMR: Eastern and Mediterrane region

\section{Acknowledgements}

This cohort study was conducted at the Isfahan cardiovascular research institute (ICRI), affiliated with the Isfahan University of Medical Sciences (IUMS). We are grateful to the ICRC team, Isfahan Provincial Health Center, Najaf-Abad Health Office, and Arak University of Medical Sciences. The authors gratefully acknowledge the contribution of all participants. The research leading to these results has also received funding from the European Union's Horizon 2020 research and innovation programme under the Marie Skłodowska-Curie grant agreement No 712949 (TECNIOspring PLUS) and from the Agency for Business Competitiveness of the Government of Catalonia, and the Ministry of Economy and Competitiveness (MINECO), Spain, under contract DPI2017-83989-R.

\section{Authors' contributions}

NSh drafted the manuscript and contributed to the study design. $\mathrm{RH}$ contributed to the conception, and data interpretation, and critically revised the manuscript. NM contributed to the study design, acquisition, and critically revised the manuscript. HRM contributed to the data analysis and interpretation and drafted the manuscript. MM contributed to the conception and design, analysis and interpretation, and drafted manuscript. MAM contributed to the interpretation and critically revised the manuscript. NS contributed to the conception and design, interpretation, and critically revised manuscript. All authors gave final approval and are accountable for all aspects of the work and its integrity and accuracy.

\section{Funding}

The research leading to these results has received funding from the European Union's Horizon 2020 research and innovation programme under the Marie Skłodowska-Curie grant agreement No 712949 (TECNIOspring PLUS) and from the Agency for Business Competitiveness of the Government of Catalonia, This work was supported by the Spanish Ministry of Economy and Competitiveness - Spain (DPI2017-83989-R), and Isfahan University of Medical Sciences.

\section{Availability of data and materials}

The datasets used and/or analysed during the current study are available from the corresponding author on reasonable request.

\section{Ethics approval and consent to participate}

All subjects signed the consent form. The experimental protocol was in accordance with the principles contained in the Helsinki Declaration that was reviewed and approved by the Ethics Committee of the Isfahan Cardiovascular Research Center, a WHO-collaborating centre at EMR, and Isfahan University of Medical Sciences.

\section{Consent for publication}

Not applicable.

\section{Competing interests}

The authors declare that they have no competing interests.

\section{Author details}

${ }^{1}$ School of Public Health, Isfahan University of Medical Sciences, Isfahan, Iran. ${ }^{2}$ Interventional Cardiology Research Center, Cardiovascular Research Institute, Isfahan University of Medical Sciences, Isfahan, Iran. ${ }^{3}$ Hypertension Research Center, Cardiovascular Research Institute, Isfahan University of Medical Sciences, Isfahan, Iran. ${ }^{4}$ Biomedical Engineering Department, Engineering Faculty, University of Isfahan, Isfahan, Iran. ${ }^{5}$ Department of Automatic Control, Biomedical Engineering Research Center, Universitat Politècnica de Catalunya, BarcelonaTech (UPC), Barcelona, Spain. ${ }^{6}$ Pediatric Cardiovascular Research Center, Cardiovascular Research Institute, Isfahan University of Medical Sciences, Isfahan, Iran. ${ }^{7}$ Biomedical Research Networking Center in Bioengineering, Biomaterialsand Nanomedicine (CIBER-BBN), Barcelona, Spain. ${ }^{8}$ Isfahan Cardiovascular Research Center, Cardiovascular Research Institute, Isfahan University of Medical Sciences, Isfahan, Iran. ${ }^{9}$ School of Population and Public Health, Faculty of Medicine, University of British Columbia, Vancouver, Canada.

Received: 19 June 2020 Accepted: 17 August 2020

Published online: 05 September 2020

\section{References}

1. World Health Organization. World health statistics overview 2019: monitoring health for the SDGs, sustainable development goals. Geneva: World Health Organization; 2019. https://apps.who.int/iris/handle/1 0665/311696.

2. World Health Organization. World health statistics 2018: monitoring health for the SDGs, sustainable development goals. Geneva: World Health Organization; 2018. p. 300.

3. Lozano R, Naghavi M, Foreman K, Lim S, Shibuya K, Aboyans V, et al. Global and regional mortality from 235 causes of death for 20 age groups in 1990 and 2010: a systematic analysis for the global burden of disease study 2010. Lancet. 2012;380(9859):2095-128.

4. Zibaeenezhad MJ, Ghaem H, Parsa N, Sayadi M, Askarian M, Kasaei M, et al. Analysing cardiovascular risk factors and related outcomes in a middle-aged to older adults population in Iran: a cohort protocol of the shiraz heart study (SHS). BMJ Open. 2019;9(4):e026317.

5. Sarrafzadegan N, Mohammmadifard N. Cardiovascular disease in Iran in the last 40 years: prevalence, mortality, morbidity, challenges and strategies for Cardiovascular prevention. Arch Iran Med. 2019;22(4):204-10 Available from: http://www.aimjournal.ir/Article/aim-5445.

6. Feigin VL, Norrving B, Mensah GA. Global burden of stroke. Circ Res. 2017; 120(3):439-48

7. World Health Organization. Prevention of cardiovascular disease : guidelines for assessment and management of total cardiovascular risk. Geneva: World Health Organization; 2007. https://apps.who.int/iris/handle/10665/43685. 
8. Pirani N, Khiavi FF. Population attributable fraction for Cardiovascular diseases Risk Factors in selected countries: a comparative study. Mater Sociomed. 2017;29(1):35-9.

9. Song $\mathrm{H}$, Fang F, Arnberg FK, Mataix-Cols D, de la Cruz L, Almqvist C, et al. Stress related disorders and risk of cardiovascular disease: population based, sibling controlled cohort study. BMJ. 2019;365:1-10. Available from: https:// www.bmj.com/content/365/bmj.l1255.

10. Wang J, Tillin T, Hughes AD, Chaturvedi N. Associations between family history and coronary artery calcium and coronary heart disease in British Europeans and South Asians. Int J Cardiol. 2019;300:39-42.

11. LaCroix AZ, Bellettiere J, Rillamas-Sun E, Di C, Evenson KR, Lewis CE, et al. Association of light physical activity measured by accelerometry and incidence of coronary heart disease and cardiovascular disease in older women. JAMA Netw Open. 2019;2(3):e190419.

12. Toth PP, Granowitz C, Hull M, Liassou D, Anderson A, Philip S. High triglycerides are Associated With increased Cardiovascular events, medical costs, and resource use: a real-world administrative claims analysis of statintreated patients With high residual Cardiovascular Risk. J Am Heart Assoc. 2018;7(15):e008740.

13. Peters SAE, Muntner P, Woodward M. Sex differences in the prevalence of and trends in, cardiovascular risk factors, treatment, and control in the United States, 2001 to 2016. Circulation. 2019;139(8):1025-35.

14. Mohammadifard N, Talaei M, Sadeghi M, Oveisegharan S, Golshahi J, Esmaillzadeh A, et al. Dietary patterns and mortality from cardiovascular disease: Isfahan cohort study. Eur J Clin Nutr. 2017;71(2):252.

15. Qi Y, Han X, Zhao D, Wang W, Wang M, Sun J, et al. Long-term cardiovascular risk associated with stage 1 hypertension defined by the 2017 acc/aha hypertension guideline. J Am Coll Cardiol. 2018;72(11):120110.

16. Yazdani A, Yazdani A, Samiei A, Boerwinkle E. Generating a robust statistical causal structure over 13 cardiovascular disease risk factors using genomics data. J Biomed Inform. 2016;60:114-9 Available from: http://www. sciencedirect.com/science/article/pii/S1532046416000137.

17. Seixas AA, Vallon J, Barnes-Grant A, Butler M, Langford AT, Grandner MA et al. Mediating effects of body mass index, physical activity, and emotional distress on the relationship between short sleep and cardiovascular disease. Medicine (Baltimore). 2018;97(37):e11939.

18. Scott-Storey KA, Hodgins M, Wuest J. Modeling lifetime abuse and cardiovascular disease risk among women. BMC Cardiovasc Disord. 2019; 19(1):224.

19. Fong TCT, Ho RTH. Factor analyses of the hospital anxiety and depression scale: a Bayesian structural equation modeling approach. Qual Life Res. 2013;22(10):2857-63.

20. Kerkhof GF, Duivenvoorden HJ, Leunissen RWJ, Hokken-Koelega ACS. Pathways leading to atherosclerosis: a structural equation modeling approach in young adults. Hypertension. 2011;57(2):255-60

21. Goong H, Ryu S, Xu L. A structural model of health behavior modification among patients with cardiovascular disease. Appl Nurs Res. 2016;29:70-5.

22. Sarrafzadegan N, Talaei M, Sadeghi M, Kelishadi R, Oveisgharan S, Mohammadifard N, et al. The Isfahan cohort study: rationale, methods and main findings. J Hum Hypertens. 2011;25(9):545-53.

23. World Health Organization. WHO draft protocol and manual of operations population survey for cardiovascular disease risk factors in the eastern Mediterranean region. Alexandria World Heal Organ. 1995;35.

24. World Health Organization. Obesity: preventing and managing the global epidemic. Geneva: World Health Organization; 2000.

25. Esteghamati A, Ashraf H, Rashidi A, Meysamie A. Waist circumference cut-off points for the diagnosis of metabolic syndrome in Iranian adults. Diabetes Res Clin Pract. 2008:82(1):104-7.

26. Working group on risk and blood pressure. An epidemiological approach to describing risk associated with blood pressure levels. Hypertension. 1985;7: 641-51.

27. Mohammadifard N, Sajjadi F, Maghroun M, Alikhasi H, Nilforoushzadeh F, Sarrafzadegan N. Validation of a simplified food frequency questionnaire for the assessment of dietary habits in Iranian adults: Isfahan healthy heart program, Iran. ARYA Atheroscler. 2015;11(2):139.

28. Talaei M, Rabiei K, Talaei Z, Amiri N, Zolfaghari B, Kabiri P, et al. Physical activity, sex, and socioeconomic status: a population based study. ARYA Atheroscler. 2013;9(1):51.

29. Goldberg DP, Hillier VF. A scaled version of the general health questionnaire. Psychol Med. 1979;9(1):139-45.
30. World Health Organization. Development of the World Health Organization WHOQOL-BREF quality of life assessment. Psychol Med. 1998;28(3):551-8.

31. Nejat S, Montazeri A, Holakouie Naieni K, Mohammad K, Majdzadeh SR. The World Health Organization quality of Life (WHOQOL-BREF) questionnaire: Translation and validation study of the Iranian version. J Sch Public Heal Inst Public Heal Res. 2006;4(4) Available from: http://sjsph.tums.ac.ir/article-1-187en.html.

32. World Health Organization. Division of Mental Health. WHOQOL-BREF: introduction, administration, scoring and generic version of the assessment: field trial version, December 1996. Geneva: World Health Organization; 1996. https://apps.who.int/iris/handle/10665/63529.

33. Usefy AR, Ghassemi GR, Sarrafzadegan N, Mallik S, Baghaei AM, Rabiei K. Psychometric properties of the WHOQOL-BREF in an Iranian adult sample. Community Ment Health J. 2010;46(2):139-47.

34. Lomax RG, Schumacker RE. A beginner's guide to structural equation modeling. psychology press; 2004.

35. Song $X-Y$, Lee $S-Y$. A tutorial on the Bayesian approach for analysing structural equation models. J Math Psychol. 2012;56(3):135-48 Available from: http://www.sciencedirect.com/science/article/pii/S0022249612000065.

36. Lee S-Y, Song X-Y. Basic and advanced Bayesian structural equation modeling: With applications in the medical and behavioral sciences: Wiley; 2012. https://onlinelibrary.wiley.com/doi/book/10.1002/9781118358887. Print ISBN:9780470669525 |Online ISBN:9781118358887 https://doi.org/10.1002/ 9781118358887

37. Sorlie P, Wei GS. Population-based cohort studies: still relevant? J Am Coll Cardiol. 2011;58(19):2010-3.

38. Pencina MJ, Navar AM, Wojdyla D, Sanchez RJ, Khan I, Elassal J, et al. Quantifying importance of major risk factors for coronary heart disease. Circulation. 2019;139(13):1603-11.

39. Akhuemonkhan E, Lazo M. Association between family history of diabetes and cardiovascular disease and lifestyle risk factors in the United States population: the 2009--2012 National Health and Nutrition Examination Survey. Prev Med (Baltim). 2017;96:129-34.

40. Gregson J, Kaptoge S, Bolton T, Pennells L, Willeit P, Burgess S, et al. Cardiovascular Risk Factors Associated With Venous Thromboembolism. JAMA Cardiol. 2019;4(2):163-173. Available from: https://doi.org/https://doi. org/10.1001/jamacardio.2018.4537.

41. Roy SS, Foraker RE, Girton RA, Mansfield AJ. Posttraumatic stress disorder and incident heart failure among a community-based sample of US veterans. Am J Public Health. 2015;105(4):757-63.

42. Sumner JA, Kubzansky LD, Kabrhel C, Roberts AL, Chen Q, Winning A, et al. Associations of trauma exposure and posttraumatic stress symptoms with venous thromboembolism over 22 years in women. J Am Heart Assoc. 2016;5(5):e003197.

43. Burg MM, Brandt C, Buta E, Schwartz J, Bathulapalli H, Dziura J, et al. Risk for incident hypertension Associated with PTSD in military veterans, and the effect of PTSD treatment. Psychosom Med. 2017;79(2):181.

44. Ding W, Li T, Su Q, Yuan M, Lin A. Integrating factors associated with hypertensive patients' self-management using structural equation modeling: a cross-sectional study in Guangdong, China. Patient Prefer Adherence. 2018:12:2169-78.

45. Uijl A, Koudstaal S, Direk K, Denaxas S, Groenwold RHH, Banerjee A, et al. Risk factors for incident heart failure in age- and sex-specific strata: a population-based cohort using linked electronic health records. Eur J Heart Fail 2019;21(10):1197-1206. Available from: https://onlinelibrary.wiley.com/ doi/abs/https://doi.org/10.1002/ejhf.1350.

46. Jung M-H, Yi S-W, An SJ, Yi J-J. Age-specific associations between systolic blood pressure and cardiovascular mortality. Heart. 2019;105(14):1070-7.

47. Miller RG, Costacou T, Orchard TJ. Risk Factor Modeling for Cardiovascular Disease in Type 1 Diabetes in the Pittsburgh Epidemiology of Diabetes Complications (EDC) Study: A Comparison With the Diabetes Control and Complications Trial/Epidemiology of Diabetes Interventions and Complication. Diabetes. 2019;68(2):409 LP-419 Available from: http:// diabetes.diabetesjournals.org/content/68/2/409.abstract.

48. Owusu Adjah ES, Ray KK, Paul SK. Ethnicity-specific association of BMI levels at diagnosis of type 2 diabetes with cardiovascular disease and all-cause mortality risk. Acta Diabetol. 2019;56(1):87-96. Available from: https://doi. org/https://doi.org/10.1007/s00592-018-1219-7.

49. Bergman E, Loyttyniemi E, Rautava P, Veromaa V, Korhonen PE. Ideal cardiovascular health and quality of life among Finnish municipal employees. Prev Med Rep. 2019;15:100922. 
50. Moonesinghe R, Yang Q, Zhang Z, Khoury MJ. Prevalence and Cardiovascular health impact of family history of premature heart disease in the United States: analysis of the National Health and nutrition examination survey, 2007-2014. J Am Heart Assoc. 2019 Jul;8(14):e012364.

51. Gao F, Liu X, Wang X, Chen S, Shi J, Zhang Y, et al. Changes in Cardiovascular health status and the Risk of new-onset hypertension in Kailuan cohort study. PLoS One. 2016;11(7):1-13. Available from: https://doi. org/https://doi.org/10.1371/journal.pone.0158869.

52. Brunström M, Carlberg B. Association of Blood Pressure Lowering With Mortality and Cardiovascular Disease across Blood Pressure Levels: a systematic review and meta-analysis. JAMA Intern Med. 2018;178(1):28-36. Available from: https://doi.org/https://doi.org/10.1001/jamainternmed.2017. 6015.

53. Bundy JD, Li C, Stuchlik P, Bu X, Kelly TN, Mills KT, et al. Systolic blood pressure reduction and Risk of Cardiovascular disease and mortality: a systematic review and network meta-analysis. JAMA Cardiol. 2017;2(7):775781. Available from: https://doi.org/https://doi.org/10.1001/jamacardio.2017. 1421.

54. Ettehad D, Emdin CA, Kiran A, Anderson SG, Callender T, Emberson J, et al. Blood pressure lowering for prevention of cardiovascular disease and death: a systematic review and meta-analysis. Lancet. 2016;387(10022):957-67 Available from: http://www.sciencedirect.com/science/article/pii/S01406 73615012258 .

55. Yusuf S, Joseph P, Rangarajan S, Islam S, Mente A, Hystad P, et al. Modifiable risk factors, cardiovascular disease, and mortality in 155722 individuals from 21 high-income, middle-income, and low-income countries (PURE): a prospective cohort study. Lancet. 2020;395(10226):795-808 Available from: http://www.sciencedirect.com/science/article/pii/S0140673619320082.

56. Kinoshita M, Yokote $K$, Arai H, lida M, Ishigaki Y, Ishibashi S, et al. Japan Atherosclerosis Society (JAS) guidelines for prevention of atherosclerotic cardiovascular diseases 2017. J Atheroscler Thromb. 2018;25(9):846-984.

57. Fogacci F, Cicero AFG, D'Addato S, D'Agostini L, Rosticci M, Giovannini M, et al. Serum lipoprotein(a) level as long-term predictor of cardiovascular mortality in a large sample of subjects in primary cardiovascular prevention: data from the Brisighella heart study. Eur J Intern Med. 2017;37:49-55 Available from: http://www.sciencedirect.com/science/article/pii/S095362 0516302588

58. Boen C, Yang YC. Chapter 25 - Social Relationships and Cardiovascular Health: Underlying Mechanisms, Life Course Processes, and Future Directions. In: Watson RR, Zibadi S, editors. Lifestyle in Heart Health and Disease. Cambridge: Academic Press; 2018. p. 291-6. Available from: http:// www.sciencedirect.com/science/article/pii/B9780128112793000252.

59. Xia N, Li H. Loneliness, social isolation, and cardiovascular health. Antioxid Redox Signal. 2018;28(9):837-51.

60. Lee I-M, Shiroma EJ, Evenson KR, Kamada M, LaCroix AZ, Buring JE. Accelerometer-measured physical activity and sedentary behavior in relation to all-cause mortality: the Women's health study. Circulation. 2018;137(2): 203-5.

61. Piercy KL, Troiano RP, Ballard RM, Carlson SA, Fulton JE, Galuska DA, et al. The physical activity guidelines for Americans. Jama. 2018;320(19):2020-8.

62. Salas-Salvadó J, Becerra-Tomás N, García-Gavilán JF, Bulló M, Barrubés L. Mediterranean diet and Cardiovascular disease prevention: what do we know? Prog Cardiovasc Dis. 2018;61(1):62-7 Available from: http://www. sciencedirect.com/science/article/pii/S0033062018300744.

63. Mohammadifard N, Nazem M, Naderi G-A, Saghafian F, Sajjadi F, Maghroon $M$, et al. Effect of hydrogenated, liquid and ghee oils on serum lipids profile. ARYA Atheroscler. 2010;6(1):16.

64. Martínez-González MA, Gea A, Ruiz-Canela M. The Mediterranean diet and Cardiovascular health. Circ Res. 2019;124(5):779-98.

65. Garshick MS, Vaidean GD, Vani A, Underberg JA, Newman JD, Berger JS, et al. Cardiovascular Risk factor control and lifestyle Factors in young to middle-aged adults with newly diagnosed obstructive coronary artery disease. Cardiology 2019;142(2):83-90. Available from: https://www.karger. com/DOI/https://doi.org/10.1159/000498891.

66. Mamudu HM, Paul TK, Wang L, Veeranki SP, Panchal HB, Alamian A, et al. Association Between Multiple Modifiable Risk Factors of Cardiovascular Disease and Hypertension among Asymptomatic Patients in Central Appalachia. South Med J. 2017;110(2):90-96. Available from: https://doi. org/https://doi.org/10.14423/SMJ.0000000000000602.
67. Beaglehole R, Bonita R, Horton R, Adams C, Alleyne G, Asaria P, et al. Priority actions for the non-communicable disease crisis. Lancet. 2011;377(9775): 1438-47.

68. Benjamin EJ, Muntner $P$, Bittencourt MS. Heart disease and stroke statistics2019 update: a report from the American Heart Association. Circulation. 2019;139(10):e56-e528.

69. Cioni G, Abouzaki NA, Jovin IS. Acute Coronary Syndrome: Thrombotic Lesions in Patients with Unstable Angina. Cardiovasc Thrombus. 1st ed. Cambridge: Elsevier; 2018. p. 147-60.

70. Rücker V, Keil U, Fitzgerald AP, Malzahn U, Prugger C, Ertl G, et al. Predicting 10-year risk of fatal cardiovascular disease in Germany: an update based on the score-Deutschland risk charts. PLoS One. 2016;11(9):e0162188. https:// doi.org/10.1371/journal.pone.0162188.

71. Cicero AFG, Fogacci F, Tocci G, Ventura F, Presta V, Grandi E, et al. Awareness of major cardiovascular risk factors and its relationship with markers of vascular aging: data from the Brisighella heart study. Nutr Metab Cardiovasc Dis. 2020;30(6):907-14 Available from: http://www.sciencedirect. com/science/article/pii/S0939475320300739.

\section{Publisher's Note}

Springer Nature remains neutral with regard to jurisdictional claims in published maps and institutional affiliations.
Ready to submit your research? Choose BMC and benefit from:

- fast, convenient online submission

- thorough peer review by experienced researchers in your field

- rapid publication on acceptance

- support for research data, including large and complex data types

- gold Open Access which fosters wider collaboration and increased citations

- maximum visibility for your research: over $100 \mathrm{M}$ website views per year

At BMC, research is always in progress.

Learn more biomedcentral.com/submissions 